\title{
Dental Caries Experience and Total Sugar Consumption-Analysis of NHANES 2011-2012
}

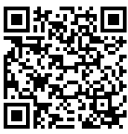

\author{
Asma Muzaffar* \\ Department of Epidemiology, NYU College of Dentistry, USA
}

Submission: December 16, 2017 ; Published: March 23, 2018

*Corresponding author: Asma Muzaffar, Department of Epidemiology, Adjunct Assistant Professor, NYU College of Dentistry, USA, Email:am8670@nyu.edu

Abstract

Background: Dental caries is the most prevalent chronic disease amongst adults. The onset of dental caries experience is multi factorial, with dietary habits, dental hygiene and access to preventive services including fluoridated water being necessary components of the causal pie. This paper investigates whether total sugar consumed in grams or percentage of total daily energy intake, changed the odds of dental caries experience adjusted for race, gender, family poverty income ratio and source of water.

Methods: The National Health and Nutrition Examination Surveys (NHANES) for calendar years 2011-2012 was used with accompanying weights to determine the prevalence of dental caries experience, the mean incidence of dental decay, the percentage of energy consumed from sugars. Multilevel Multivariable logistic regressions were used for statistical analysis. Odds Ratios (Adjusted and unadjusted) were reported with $95 \%$ Confidence Intervals.

Results: In 2011-2012 69.03\% of the US population had at least one caries experience, defined as the presence of at least one tooth with untreated decay, or restored (fillings, fixed and partial prosthesis due to dental disease) or missing due to dental disease. When stratified by age, dental caries experience defined as above was most prevalent amongst young adults and adolescents with prevalence of 70.64 amongst 11-20 year olds. The mean total sugar consumption was 115.34 grams (CI 112.86117 .81 ) and the mean daily energy consumption from sugar was $23.96 \%$ (CI 23.6724 .27 ). Sub population analysis of dental caries experience amongst all ages from 0 up to 35 years of age done revealed a caries experience prevalence of $69.74 \%$ in all ages up to 35 years of age.

The median consumption of total daily energy intake from sugars was $23.3 \%$ amongst Americans in 2011-2012. The mean teeth to be involved with dental caries experience were (mean dmft score) 7.24 (95\%CL 7.24,7,76).

Chi square test was not significant for categories of sugar consumption and caries experience $(0.14,0.19,0.57)$ There was no change in the odds of dental caries experience with change in the total sugar consumption in grams $(0.99,1.00)$ and change in the amount of sugar consumed as a percentage of total daily calorie intake $(0.95-1.3,0.93-1.4,0.86-1.2)$.

Conclusion: The above findings support previous literature, that indeed it is not the net sugar consumption that influences the caries experience; rather the dietary component of the causal pie of caries experience needs to be exhaustively studied, in light of recent advancements in dental public health.

\section{Introduction}

The first ever US Surgeon General report on Oral Health, themed around oral health as a reflection of general health and well-being was published at the onset of the twenty first century. From sealant programs to water fluoridation, dental profession has been at the forefront of decreasing the population burden of dental disease via public health measures. Yet, dental disease amongst permanent teeth is the single most prevalent condition worldwide affecting 2.5 billion people (prevalence $34.1 \%$ for all ages).

Dental decay is understood to be multi factorial with dietary habits as a cornerstone of the onset of disease. The breakdown of cariogenic food types, decreasing the ph of saliva, by the energized oral micro biome, is the ignition for caries process. Overtime in the presence of poor oral hygiene and absence of fluoride treatment or sealants, this caries process often concludes into dental disease. Refined carbohydrates and sugars are typically considered amongst the cariogenic food types, which decrease the ph around teeth, lending them susceptible to caries process.

However, systematic review by Burt \& Pai [1] found a weak association between caries risk and sugar consumption. Further the review concluded with the following remarks, "in modern age, with fluoride exposure, the relationship between caries risk and sugar may not be as strong as it used to be". The authors also suggested that controlling for sugar is justifiable, but it may not be the most important preventive measure in today's period. Recent research published in British Journal suggests reducing intake of sugar to $3 \%$ to reduce dental caries [2]. The aim of this paper is 
to measure the association between dental caries experience and percentage of total daily calories consumed from sugar in the NHANES dataset 2011-2012.

\section{Methods}

\section{Data Source}

Data were obtained from The National Health and Nutrition Examination Survey (NHANES) for the years 2011-2012. NHANES is a population based survey, in which the participants are selected using random sampling method, representing non institutionalized US population.

\section{Study Population}

The study population included 8956 participants aged 0 years and older

\section{Variable Classification}

Occupant characteristics

\section{Demographics}

Age was analyzed both as a continuous and categorical variables. As a categorical variable age was categorized into six categories with ages 0 and 13 as one category, ages 13 and 20 as second category, ages 20 and 35 as third category, ages 35 to 55 as fourth category, ages 55 to 65 as fifth category and over age 65 as sixth category.

a) Gender was characterized as male or female as provided in (NHANES).

b) Race was characterized as Mexican American, Other Hispanic, Non Hispanic Black, Non Hispanic White and Other Race.

Dental Caries experience was dichotomized as:

1. Presence of caries experience

2. Absence of caries experience.

Caries experience was calculated as sum of all the decay, missing due to dental disease, missing due to dental disease and replaced with prosthesis (partial or fixed) and filled teeth. A sum of greater than or equal to 1 was categorized as presence of dental caries experience and less than 1 was categorized as absence of caries. The variables (OHX02CTC...OHX31CTC) from the oral health examination dataset were used to calculate the above information. Dental disease count was calculated as a sum of decayed, missing due to dental disease, missing due to dental disease and replaced by prosthesis (partial or fixed) and filled teeth.

\section{Dietary Variables}

The dietary variables were retrieved from dietary intake data of NHANES. Dietary intake data of nhanes, is 24 hour dietary recall of the types and amounts of foods and beverages (including all types of water) consumed. The first dietary recalls interview is collected in person in the Mobile Examination Center and the second interview is collected 3 to 10 days later via telephone.
Interviews for children 6 years of age and younger was conducted using a proxy, 6 to 8 years of age with the proxy and child present to assist in reporting, 9 to 11 with child and an adult able to assist and children 12 years of age or older answered for themselves).

Below is brief description of the variables retrieved from NHANES:

a) Total Sugar consumption in grams: Retrieved from total nutrient intake files file of the dietary component of NHANES.

b) Source of water: (Community supply, Well or rain cistern, spring, don't drink tap water) was retrieved from the total nutrient intake file of the dietary component of NHANES.

c) Total energy consumed: was retrieved from the total nutrient intake records of the dietary component of NHANES.

d) Percentage of daily calories consumed from sugar: was calculated in SAS using the formula total sugar consumption in grams $* 4$ /Total energy consumed $* 100$.

e) SugarP: A categorical variable depicting the percentage of daily calories consumed from sugar was created after retrieving the distribution of percentage of daily calories consumed from sugar (Table 1). The categories were created to stratify relationship of percentage of sugars consumed from calories and was defined as 0 if percentage of daily calories consumed from sugar was between 0 and 17, 1 if percentage of daily calories consumed from sugar was between 17 and 24, 2 if percentage of daily calories consumed from sugar was between 24 and 30 and 3 if percentage of daily calories consumed from sugar was greater than 30 .

f) Distribution of PCTS1 using Proc Univariate: SugarP categorized based on below distribution

(Table 1)

Table 1.

\begin{tabular}{|c|c|}
\hline \multicolumn{2}{|c|}{ Quantiles (Definition 5) } \\
\hline Quantile & Estimate \\
\hline $100 \%$ Max & 89.78378 \\
\hline $99 \%$ & 53.44556 \\
\hline $95 \%$ & 42.13149 \\
\hline $90 \%$ & 37.57746 \\
\hline $75 \%$ Q3 & 30.30791 \\
\hline $50 \%$ Median & 23.30241 \\
\hline $25 \%$ Q1 & 16.98946 \\
\hline $10 \%$ & 11.37837 \\
\hline $5 \%$ & 8.24913 \\
\hline $1 \%$ & 3.68144 \\
\hline $0 \%$ Min & 0.00000 \\
\hline
\end{tabular}


g) Compare food consumed yesterday to usual: retrieved from the total nutrient intake records of the dietary component of NHANES. Analysis was also done on subset of population with values indicating food consumed yesterday was usual.

The dietary recall of sugar was lower on the second dietary recall (over the phone) p value $<.0001$ calculated using proc t test.

h) Compare food consumed yesterday to usual: Analysis was also done on subset of population with values of 2 which indicated food consumed yesterday was usual.

\section{Statistical analysis}

The associations of dental caries experience with total sugar consumption as categorical variables depictive of the percentage of daily calories consumption was analyzed using multilevel logistic regressions. SAS Survey logistics was used to generate Odds Ratios with 95\% Confidence Intervals. Subpopulation analysis of weighted data was also done for the participants, who recorded that their 24-hour consumption of dietary intake as recorded in the dietary component of NHANES survey was depictive of their usual intake of nutrients. Adjusted and unadjusted ratios were reported. Final models included, age, gender, race, poverty income ratio and source of water. All analysis was conducted in SAS 9.4.

\section{Results}

The study population consists of 8956 adults aged 0 to 150 years. The prevalence of dental caries experience was $69.03 \%$ amongst the weighted population. Young adolescents and adults (11-20 years) had the most caries prevalence, $70.64 \%$.

Since, prevalence of periodontal disease is historically rare for infants, children and adults up to 35 years of age, Subpopulation analysis of dental caries experience amongst all ages from 0 up to 35 years of age done. This revealed a caries experience prevalence of $69.74 \%$ in all ages up to 35 years of age.

The mean dmft score was 7.24 (95\%CL 7.24,7,76). The mean dmft was lowest for Non Hispanic White (7.24) and highest for Other Race (7.78), followed by Mexican American (7.77), Non Hispanic Black (7.54) and Other Hispanics (7.41).

The mean DMFT amongst ages 0-35 years was not significant with changes in the values of total daily caloric intake from sugar ranging from 7.24 to 7.84 and overlapping confidence limits.

The plotting of data using sub sampling did not reveal a linear relationship between sugar consumption in grams (and percentage of daily calories from sugar) and dental disease count. The adjusted Odds ratio's (0.96 1.39), (0.93 1.4) (0.87 1.23) and unadjusted Odds Ratio's (0.75 1.20), (0.81 1.15), (0.84 1.29) were not significant for changes in sugar consumption as percentage of daily calories consumed from sugar and dental caries experience for values of percentage of daily calories consumed from sugar as categorical variable, using logistic regression (Table 2a-2d).
Table 2a.

\begin{tabular}{|l|c|}
\hline \multicolumn{2}{|c|}{ Any Dental Caries Experience (2011-2012) } \\
\hline Caries Experience (+) & $19,420,0087(69.03 \%)$ \\
\hline Caries Experience (-) & $8,723,5737(30.99 \%)$ \\
\hline
\end{tabular}

Table 2b.

\begin{tabular}{|c|c|c|}
\hline Age & Caries Experience(+) & Caries Experience(-) \\
\hline 0-2 years & $4398395(68.17 \%)$ & $2053257(31.82 \%)$ \\
\hline 2-5 years & $10916094(68.82 \%)$ & $4945680(31.18 \%)$ \\
\hline 6-10 years & $13493861(70.34 \%)$ & $5689947(29.66 \%)$ \\
\hline 11 -20 years & $28222809(70.64 \%)$ & $11729373(29.36 \%)$ \\
\hline $21-30$ years & $25951447(69.32 \%)$ & $11487154(30.68 \%$ \\
\hline $31-50$ years & $52030552(69.41 \%)$ & $22925995(30.58 \%)$ \\
\hline$>50$ years & $59186929(67.57 \%)$ & $28404331(32.43 \%)$ \\
\hline
\end{tabular}

Table 2c.

\begin{tabular}{|c|c|}
\hline Disease Up to 35 years & Prevalence \\
\hline Caries Experience $(+)$ & $932,43713(69.74 \%)$ \\
\hline Caries Experience $(-)$ & $404,39389(30.25 \%)$ \\
\hline Total & $1,336,83102$ \\
\hline
\end{tabular}

Table 2d.

\begin{tabular}{|c|c|c|c|}
\hline \multicolumn{3}{|c|}{ Unadjusted Odds Ratio Estimates } \\
\hline Effect & $\begin{array}{c}\text { Point } \\
\text { Estimate }\end{array}$ & $\begin{array}{c}\mathbf{9 5 \%} \text { Wald } \\
\text { Confidence Limits }\end{array}$ \\
\hline $\begin{array}{c}\text { sugarp 1 vs 0 p } \\
\text { value (0.6) }\end{array}$ & 0.950 & 0.75 & 1.20 \\
\hline $\begin{array}{c}\text { sugarp 2 vs 0 p } \\
\text { value (0.6) }\end{array}$ & 0.965 & 0.81 & 1.15 \\
\hline $\begin{array}{c}\text { sugarp 3 vs 0 p } \\
\text { value (0.7) }\end{array}$ & 1.038 & 0.84 & 1.29 \\
\hline
\end{tabular}

Adjusted OR, for Race, Gender, Povertyx and Source of Water

(Table 3a-3d)

Table 3a.

\begin{tabular}{|c|c|c|c|c|}
\hline & $\begin{array}{c}\text { Caries } \\
\text { Experience(+) }\end{array}$ & $\begin{array}{c}\text { Caries } \\
\text { Experience(-) }\end{array}$ & OR & p-value \\
\hline $\begin{array}{c}\text { Sugarp0 } \\
\text { (0-17\%dailycal } \\
\text { from sugar) }\end{array}$ & $\begin{array}{c}57393775 \\
(19.56 \%)\end{array}$ & $\begin{array}{c}25637952 \\
(8.74 \%)\end{array}$ & $\begin{array}{l}\text { Ref } \\
\text { group }\end{array}$ & \\
\hline $\begin{array}{l}\text { Sugarp } 1 \text { (17- } \\
24 \% \text { dailycal } \\
\text { from sugar) }\end{array}$ & $\begin{array}{l}60801364 \\
(20.72 \%)\end{array}$ & $\begin{array}{c}23620067 \\
(8.05 \%)\end{array}$ & $\begin{array}{l}0.96 \\
1.39\end{array}$ & 0.14 \\
\hline $\begin{array}{l}\text { Sugarp } 2 \text { (24- } \\
30 \% \text { dailycal } \\
\text { from sugar) }\end{array}$ & $\begin{array}{l}42396484 \\
(14.45 \%)\end{array}$ & $\begin{array}{c}16647745 \\
(5.67 \%)\end{array}$ & $\begin{array}{l}0.93 \\
1.40\end{array}$ & 0.19 \\
\hline $\begin{array}{c}\text { Sugarp } 3 \\
\text { (>30\% dailycal } \\
\text { from sugar) }\end{array}$ & $\begin{array}{c}46950756( \\
16.00 \%)\end{array}$ & $\begin{array}{c}19926160 \\
(6.79 \%)\end{array}$ & $\begin{array}{l}0.87 \\
1.27\end{array}$ & 0.57 \\
\hline
\end{tabular}


Table 3b.

\begin{tabular}{|c|c|c|c|c|}
\hline Race & $\begin{array}{c}\text { Caries } \\
\text { Experience } \\
(+)\end{array}$ & $\begin{array}{c}\text { Caries } \\
\text { Experience } \\
(-)\end{array}$ & $\begin{array}{c}\text { OR Ref NH } \\
\text { White }\end{array}$ & p-value \\
\hline $\begin{array}{c}\text { Mexican } \\
\text { American }\end{array}$ & $\begin{array}{c}18837476 \\
(6.69 \%)\end{array}$ & $\begin{array}{c}7956499 \\
(2.82 \%)\end{array}$ & $0.79,1.25$ & 0.96 \\
\hline $\begin{array}{c}\text { Other } \\
\text { Hispanic }\end{array}$ & $\begin{array}{c}14059548 \\
(4.99 \%)\end{array}$ & $\begin{array}{c}5537555 \\
(1.96 \%)\end{array}$ & $1.001,1.34$ & 0.04 \\
\hline $\begin{array}{c}\text { Non- } \\
\text { Hispanic } \\
\text { White }\end{array}$ & $\begin{array}{c}120362186 \\
(42.77 \%)\end{array}$ & $\begin{array}{c}56987522 \\
(20.25 \%)\end{array}$ & & \\
\hline $\begin{array}{c}\text { Non- } \\
\text { Hispanic } \\
\text { Black }\end{array}$ & $\begin{array}{c}24827005 \\
(8.8 \%)\end{array}$ & $\begin{array}{c}10276708 \\
(3.65 \%)\end{array}$ & $0.85,1.30$ & 0.62 \\
\hline Other Race & $\begin{array}{c}16113871 \\
(5.72 \%)\end{array}$ & $\begin{array}{c}6477454 \\
(2.3 \%)\end{array}$ & 0.881 .394 & 0.37 \\
\hline
\end{tabular}

Table 3c.

\begin{tabular}{|c|c|c|c|c|}
\hline Race & \multicolumn{4}{|c|}{ Sugarp } \\
\hline & $\begin{array}{c}0(0-17 \% \\
\text { daily } \\
\text { cal from } \\
\text { sugar })\end{array}$ & $\begin{array}{c}1(17-24 \% \\
\text { daily } \\
\text { cal from } \\
\text { sugar })\end{array}$ & $\begin{array}{c}2(24-30 \% \\
\text { daily cal } \\
\text { from sugar })\end{array}$ & $\begin{array}{c}3(>30 \% \\
\text { daily cal } \\
\text { from sugar })\end{array}$ \\
\hline $\begin{array}{c}\text { Mexican } \\
\text { American }\end{array}$ & $\begin{array}{c}10068889 \\
(3.4 \%)\end{array}$ & $\begin{array}{c}10338536 \\
(3.5 \%)\end{array}$ & $\begin{array}{c}7358737 \\
(2.5) \%\end{array}$ & $\begin{array}{c}10204233 \\
(3.47 \%)\end{array}$ \\
\hline $\begin{array}{c}\text { Other } \\
\text { Hispanic }\end{array}$ & $\begin{array}{c}9120533 \\
(3.1 \%)\end{array}$ & $\begin{array}{c}8446942 \\
(2.87 \%)\end{array}$ & $\begin{array}{c}6447085 \\
(2.19 \%)\end{array}$ & $\begin{array}{c}7348324 \\
(2.5 \%)\end{array}$ \\
\hline $\begin{array}{c}\text { Non- } \\
\text { Hispanic } \\
\text { White }\end{array}$ & $\begin{array}{c}26468263 \\
(9.02 \%)\end{array}$ & $\begin{array}{c}27471148 \\
(1.2 \%)\end{array}$ & $\begin{array}{c}20750598 \\
(7.07 \%)\end{array}$ & $\begin{array}{c}19950176 \\
(6.8 \%)\end{array}$ \\
\hline $\begin{array}{c}\text { Non- } \\
\text { Hispanic } \\
\text { Black }\end{array}$ & $\begin{array}{c}22890562 \\
(7.8 \%)\end{array}$ & $\begin{array}{c}23700703 \\
(8.07 \%)\end{array}$ & $\begin{array}{c}14654418 \\
(4.99 \%)\end{array}$ & $\begin{array}{c}17520413 \\
(5.97 \%)\end{array}$ \\
\hline $\begin{array}{c}\text { Other } \\
\text { Race }\end{array}$ & $\begin{array}{c}14483480 \\
(4.9 \%)\end{array}$ & $\begin{array}{c}14464102 \\
(4.93 \%)\end{array}$ & $\begin{array}{c}9833391 \\
(3.35 \%)\end{array}$ & $\begin{array}{c}11853771 \\
(4.04 \%)\end{array}$ \\
\hline
\end{tabular}

Table 3d.

\begin{tabular}{|c|c|c|c|}
\hline $\begin{array}{c}\text { Caries } \\
\text { Experience(+) }\end{array}$ & $\begin{array}{c}\text { Caries } \\
\text { Experience(-) }\end{array}$ & $\begin{array}{c}\text { OR } \\
(\mathbf{9 5} \% \mathbf{C I})\end{array}$ & P-VALUE \\
\hline $\begin{array}{c}74996209 \\
(25.42 \%)\end{array}$ & $\begin{array}{c}31535281 \\
(10.68 \%)\end{array}$ & 0.8561 .152 & 0.9 \\
\hline $\begin{array}{c}49928511 \\
(16.92 \%)\end{array}$ & $\begin{array}{c}20217986 \\
(6.8 \%)\end{array}$ & 0.8781 .239 & 0.6 \\
\hline $\begin{array}{c}83757590 \\
(28.39 \%)\end{array}$ & $34605389(11.7 \%)$ & Ref & \\
\hline
\end{tabular}

\section{Analysis of Dental Disease As Continuous Variable}

(Table 4a-4d, Graph 1).

Table 4a.

\begin{tabular}{|c|c|c|c|c|c|}
\hline \multicolumn{5}{|c|}{ Statistics } \\
\hline Variable & N & Mean & $\begin{array}{c}\text { Std Error } \\
\text { of Mean }\end{array}$ & \multicolumn{2}{|c|}{ 95\% CL for Mean } \\
\hline DMFT & 8169 & 7.50 & 0.123469 & 7.24224099 & 7.76323610 \\
\hline
\end{tabular}

Table 4b.

\begin{tabular}{|c|c|c|c|c|c|c|}
\hline \multicolumn{7}{|c|}{ Domain Analysis: Race/Hispanic origin } \\
\hline $\begin{array}{c}\text { Race/Hispanic } \\
\text { origin }\end{array}$ & Variable & N & Mean & $\begin{array}{c}\text { Std } \\
\text { Error of } \\
\text { Mean }\end{array}$ & \multicolumn{2}{|c|}{$\begin{array}{c}\text { 95\% CL for } \\
\text { Mean }\end{array}$} \\
\hline $\begin{array}{c}\text { Mexican } \\
\text { American }\end{array}$ & DMFT & 1111 & 7.77 & 0.249758 & 7.25 & 8.30 \\
\hline Other Hispanic & DMFT & 889 & 7.41 & 0.288870 & 6.80 & 8.02 \\
\hline $\begin{array}{c}\text { Non Hispanic } \\
\text { White }\end{array}$ & DMFT & 2496 & 7.24 & 0.308375 & 6.58 & 7.88 \\
\hline $\begin{array}{c}\text { Non Hispanic } \\
\text { Black }\end{array}$ & DMFT & 2270 & 7.55 & 0.292534 & 6.93 & 8.16 \\
\hline Other Race & DMFT & 1403 & 7.78 & 0.312174 & 7.12 & 8.44 \\
\hline
\end{tabular}

Table 4c.

\begin{tabular}{|c|c|c|c|c|c|c|}
\hline \multicolumn{6}{|c|}{ DMFT for different categories of percentage of total daily calories } \\
from sugar
\end{tabular}

Table 4d.

\begin{tabular}{|c|c|c|c|c|c|c|}
\hline \multicolumn{7}{|c|}{$\begin{array}{c}\text { DMFT for ages } 0 \text { to } 35 \text { years based on total daily calories } \\
\text { consumed from sugar }\end{array}$} \\
\hline Sugarp & Variable & N & Mean & $\begin{array}{c}\text { Std Error } \\
\text { of Mean }\end{array}$ & $\begin{array}{c}\text { 95\% CL for } \\
\text { Mean }\end{array}$ \\
\hline 0 & DMFT & 1140 & 7.59 & 0.307657 & 6.95 & 8.24 \\
\hline 1 & DMFT & 1267 & 7.84 & 0.282099 & 7.24 & 8.43 \\
\hline 2 & DMFT & 976 & 7.48 & 0.455605 & 6.52 & 8.44 \\
\hline 3 & DMFT & 1127 & 7.24 & 0.262174 & 6.68 & 7.79 \\
\hline
\end{tabular}

Using codes

/* plotting a representative subsample*/

proc surveyselect data=thesis.mastereepd 1 out=plotdata

method=pps sampsize $=\mathbf{1 3 0 0}$ seed $=\mathbf{3 4 5 2}$;

size wtdrd1;

run;

symbol1 $\mathrm{v}=$ circle $\mathrm{i}=\mathrm{r} \mathrm{c}=$ red $\mathrm{ci}=$ green $\mathrm{w}=\mathbf{2}$;

proc gplot data=plotdata;

plot pcts1*dentalDiseasecount; 
run;

proc surveyselect data=thesis.mastereepd1 out=plotdata method $=$ pps sampsize $=\mathbf{1 3 0 0}$ seed $=\mathbf{3 4 5 2}$;

size wtdrd1;

run; symbol $1 \mathrm{v}=$ circle $\mathrm{i}=\mathrm{r} \mathrm{c}=$ purple $\mathrm{ci}=$ green $\mathrm{w}=3$;

proc gplot data=plotdata;

plot Dr1tsugr*dentaldiseasecount;

run

(Graph 2)

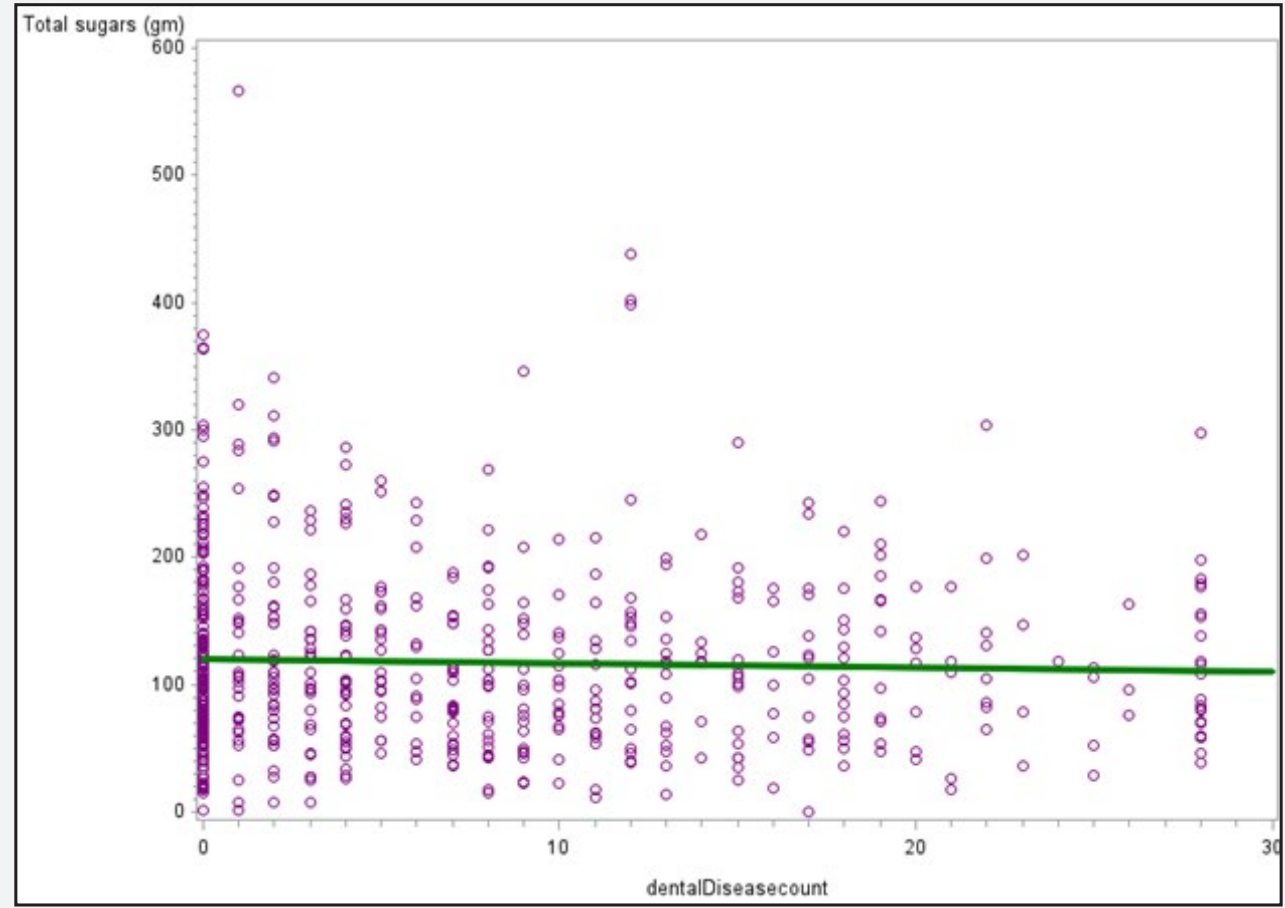

Graph 1: Graphical representation of relationship between caries experience as continuous variable (dental disease count) and total sugar consumption in grams from a subsample (1300) weighted population.

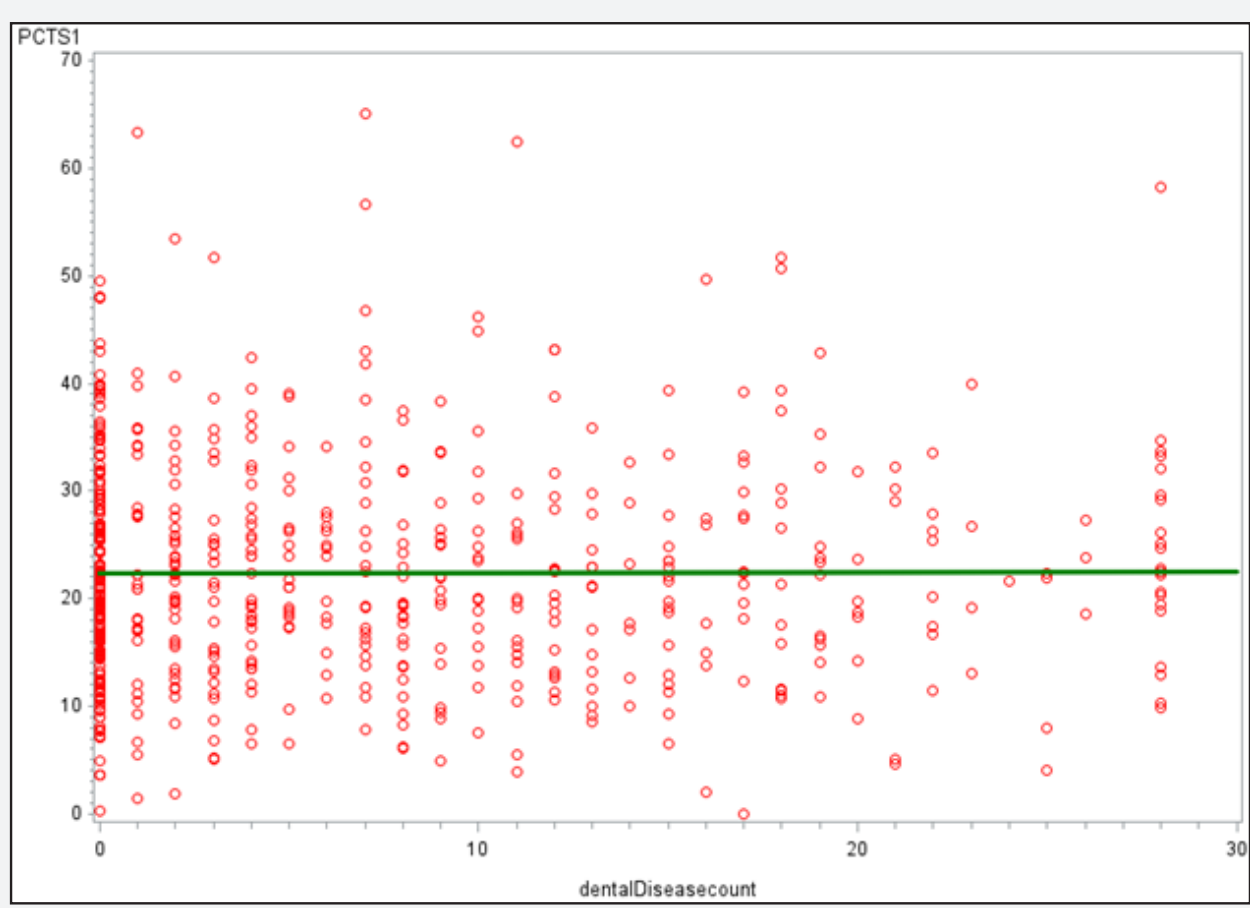

Graph 2 : Graphical representation of relationship between caries experience as continuous variable (dental disease count) and percentage of daily calories consumed from sugar from a subsample (1300) weighted population. 
Since the graphical representation of dental disease count did not exhibit a linear relationship with increasing values of total sugars in grams and percentage of total daily caloric intake from sugar, a categorical variable characterizing the severity of dental disease was created with values 0 for $\mathrm{dmft} 0,1$ for $\mathrm{dmft}$ counts1-7, value 2 for $\mathrm{dmft}$ counts between 7 to 14 , and value 3 for $\mathrm{dmft}$ counts between 14 to 28 .

The measures of association categorizing severity of dental disease count with changes in percentage of total daily caloric intake from sugar were analyzed using logistic regressions. The odds ratios were not significant for changes in percentage of daily caloric intake from sugar and severity of dental disease count adjusted for race, gender, family poverty income ratio and source of water (Table 5).

\section{Table 5.}

\begin{tabular}{|c|c|c|c|c|}
\hline \multicolumn{5}{|c|}{ Odds Ratio Estimates } \\
\hline \multirow{2}{*}{$\begin{array}{c}\text { Effect } \\
\text { sugarp } 1 \text { vs } 0\end{array}$} & \multirow{2}{*}{$\begin{array}{c}\text { Severity } \\
1\end{array}$} & \multirow{2}{*}{$\begin{array}{c}\begin{array}{c}\text { Point } \\
\text { Estimate }\end{array} \\
1.169\end{array}$} & \multicolumn{2}{|c|}{$\begin{array}{l}\text { 95\% Wald } \\
\text { Confidence } \\
\text { Limits }\end{array}$} \\
\hline & & & 1.013 & 1.350 \\
\hline sugarp 1 vs 0 & 2 & 0.964 & 0.741 & 1.254 \\
\hline sugarp 1 vs 0 & 3 & 1.315 & 1.019 & 1.698 \\
\hline sugarp 2 vs 0 & 1 & 1.215 & 0.922 & 1.600 \\
\hline sugarp 2 vs 0 & 2 & 1.079 & 0.808 & 1.441 \\
\hline sugarp 2 vs 0 & 3 & 1.061 & 0.832 & 1.354 \\
\hline sugarp 3 vs 0 & 1 & 1.152 & 0.945 & 1.403 \\
\hline sugarp 3 vs 0 & 2 & 0.880 & 0.651 & 1.188 \\
\hline sugarp 3 vs 0 & 3 & 1.044 & 0.766 & 1.424 \\
\hline Female vs Male & 1 & 0.980 & 0.813 & 1.182 \\
\hline Female vs Male & 2 & 1.073 & 0.822 & 1.401 \\
\hline Female vs Male & 3 & 0.897 & 0.711 & 1.132 \\
\hline $\begin{array}{c}\text { Mexican American vs Non } \\
\text { Hispanic White }\end{array}$ & 1 & 0.899 & 0.720 & 1.123 \\
\hline $\begin{array}{c}\text { Mexican American vs Non } \\
\text { Hispanic White }\end{array}$ & 2 & 0.948 & 0.678 & 1.325 \\
\hline $\begin{array}{c}\text { Mexican American vs Non } \\
\text { Hispanic White }\end{array}$ & 3 & 1.252 & 0.909 & 1.725 \\
\hline $\begin{array}{l}\text { Other Hispanic vs Non } \\
\text { Hispanic White }\end{array}$ & 1 & 1.229 & 0.993 & 1.521 \\
\hline $\begin{array}{l}\text { Other Hispanic vs Non } \\
\text { Hispanic White }\end{array}$ & 2 & 1.061 & 0.729 & 1.545 \\
\hline $\begin{array}{l}\text { Other Hispanic vs Non } \\
\text { Hispanic White }\end{array}$ & 3 & 1.196 & 0.977 & 1.465 \\
\hline $\begin{array}{c}\text { Non Hispanic Black vs Non } \\
\text { Hispanic White }\end{array}$ & 1 & 0.987 & 0.750 & 1.299 \\
\hline $\begin{array}{c}\text { Non Hispanic Black vs Non } \\
\text { Hispanic White }\end{array}$ & 2 & 1.144 & 0.777 & 1.682 \\
\hline $\begin{array}{c}\text { Non Hispanic Black vs Non } \\
\text { Hispanic White }\end{array}$ & 3 & 1.117 & 0.803 & 1.554 \\
\hline $\begin{array}{l}\text { Other Race vs Non Hispanic } \\
\text { White }\end{array}$ & 1 & 1.034 & 0.797 & 1.342 \\
\hline
\end{tabular}

\begin{tabular}{|c|c|c|c|c|}
\hline $\begin{array}{c}\text { Other Race vs Non Hispanic } \\
\text { White }\end{array}$ & 2 & 1.147 & 0.767 & 1.716 \\
\hline $\begin{array}{c}\text { Other Race vs Non Hispanic } \\
\text { White }\end{array}$ & 3 & 1.249 & 0.924 & 1.687 \\
\hline Water Source & 1 & 1.000 & 1.000 & 1.000 \\
\hline Water Source & 2 & 1.000 & 1.000 & 1.000 \\
\hline Water Source & 3 & 1.000 & 1.000 & 1.000 \\
\hline povertyx 1 vs 3 & 1 & 0.925 & 0.812 & 1.054 \\
\hline povertyx 1 vs 3 & 2 & 1.122 & 0.867 & 1.450 \\
\hline povertyx 1 vs 3 & 3 & 0.917 & 0.751 & 1.119 \\
\hline povertyx 2 vs 3 & 1 & 1.080 & 0.844 & 1.382 \\
\hline povertyx 2 vs 3 & 2 & 1.065 & 0.815 & 1.391 \\
\hline povertyx 2 vs 3 & 3 & 0.913 & 0.709 & 1.175 \\
\hline
\end{tabular}

\section{Discussion}

Since oral cavity is gateway to nutritional needs of human body, it is assumed that the dietary habits play an important role to maintain the integrity of hard and soft tissues of the oral cavity. However, there is evidence that the hard tissues of the oral cavity (teeth) are more influenced by local side effects related to eating habits, compared to the periodontium, which is more influenced by systemic nutritional effects than local effects.

The local side effects of dietary habits are modified by the thickness of plaque as has been shown by Stephen and Millet, and replicated by other studies who reported no acidogenic activity of carbohydrate containing food items, when no plaque was allowed to grow using ph telemetry [3].

Comparative Studies on Turkish and Moroccan immigrant children in Netherlands revealed a higher caries rates in Turkish and Moroccan children than natives, despite the former eating less cariogenic foods and more vegetables [4].

Studies on percentage of caries free children in Basel, Switzerland revealed an increase in the percentage of caries free 7 year old children from 2-3\% during world war two (limited sugar supply) to $15 \%$. However, with the introduction of fluoride tablets, fluoride toothpastes and increased awareness, education programs, the caries free percentage of 7 year old children increased to $65 \%$ despite substantial increase in sugar consumption post world war two [5].

Hence our results in this analysis examining the changes in odds of dental caries experience, or changes in the odds of severity of dental caries with the changes in total sugar consumption as percentage of daily calories intake from sugar confirms historical evidence of poor association between total sugar consumption and dental caries experience [6].

One of the main limitations of our study was not analyzing frequency of sugar consumption and thickness of plaque. The landmark Vipeholm study of the 1950's first revealed the distinction between the frequency of the sugars intake and total amount of sugar consumed and its effect on incidence of caries. An 
increase in incidence of caries was noted with increased frequency of sugar intake rather total sugar intake in the Vipeholm study [7].

The critiques of Vipeholm study include external validation as the study was conducted in mental asylum with inmates not controlled for thickness of plaque or oral hygiene. With widespread use of fluoride containing toothpastes and improved brushing habits, leading to almost no plaque in children, and better hygiene in adults, may have accounted for no change in association between dental caries experience and total sugar consumption [810]. Future studies must be done, measuring thickness of plaque and frequency of total sugar intake to further understand the associations between dental caries and dietary sugars. Limitations of this study also included recall bias in recalling dietary sugars and teeth missing due to dental caries or periodontal disease [11]. Since prevalence of periodontal disease is limited in ages below 35 years of age, we did a subpopulation analysis up to 35 years of age and found similar non significant results.

\section{Conclusion}

The above study reiterates the concluding statements of systematic review by Burt \& Pai [1]. Total Sugar consumption in grams and percentage of daily calories consumed from sugar does not change the presence or absence of dental caries experience.

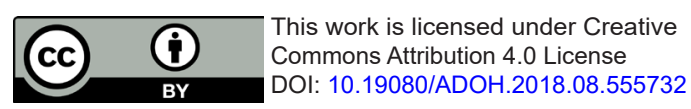

\section{References}

1. Burt BA, S Pai (2001) Sugar consumption and caries risk: a systematic review. J Dent Educ 65(10): 1017-1023.

2. Kmietowicz Z (2014) Reduce sugar intake to 3\% to protect against tooth decay, say researchers. Bmj 349: g5622.

3. Konig KG (2000) Diet and oral health. Int Dent J 50(3): 162-174.

4. (1988) bg, M.J.F.V.o. and T.e.M.k.i.n.h.n.i. NeaMana!, Amsterdam, Koninklijk Instituut voor de Tropen, pp. 272.

5. Konig KG, JM Navia (1995) Nutritional role of sugars in oral health. Am J Clin Nutr 62(1 Suppl): 275S-282S; discussion 282S-283S.

6. Harel Raviv M, M Laskaris, KS Chu (1996) Dental caries and sugar consumption into the 21st century. Am J Dent 9(5): 184-190.

7. Armfield JM, Spencer AJ, Roberts-Thomson KF, Plastow K (2013) Water fluoridation and the association of sugar-sweetened beverage consumption and dental caries in Australian children. Am J Public Health 103(3): 494-500.

8. Moynihan PJ (2005) The role of diet and nutrition in the etiology and prevention of oral diseases. Bull World Health Organ 83(9): 694-699.

9. Moynihan PJ (2002) Dietary advice in dental practice. Br Dent J 193(10): 563-568.

10. Rugg Gunn AJ (1993) Nutrition, diet and dental public health. Community Dent Health 10 Suppl 2: 47-56.

11. Nunn J (2001) Nutrition and dietary challenges in oral health. Nutrition 17(5): 426-427.

\section{Your next submission with Juniper Publishers will reach you the below assets}

- Quality Editorial service

- Swift Peer Review

- Reprints availability

- E-prints Service

- Manuscript Podcast for convenient understanding

- Global attainment for your research

- Manuscript accessibility in different formats

( Pdf, E-pub, Full Text, Audio)

- Unceasing customer service

Track the below URL for one-step submission https://juniperpublishers.com/online-submission.php 
